\title{
Lower frequency of Gaucher disease carriers among Tay-Sachs disease carriers
}

\author{
L Peleg ${ }^{1,3}$, A Frisch ${ }^{2,3}$, B Goldman ${ }^{1,3}$, M K arpaty $^{1}, \mathrm{R}$ Narinsky ${ }^{2}, \mathrm{~S}$ Bronstein ${ }^{1}$ and \\ M Frydman ${ }^{1,3}$
}

${ }^{1} \mathrm{G}$ enetic I nstitute, Sheba M edical Center, Tel H ashomer

${ }^{2}$ Felsenstein M edical R esearch Center

${ }^{3}$ Sackler Faculty of M edicine, Tel A viv U niversity, Israel

\begin{abstract}
The heterozygote frequency of Gaucher disease (GD) and Tay-Sachs disease (TSD) is distinctly high among Ashkenazi Jews (1:29 for TSD and 1:16 for GD). Two main theories have been suggested to explain this high occurrence: a founder effect with subsequent genetic drift, and a selective advantage of heterozygotes. We compared the frequency of the GD most common mutation $(1226 \mathrm{~A} \rightarrow \mathrm{G})$ among carriers of the common TSD mutation (+1277 TATC) with the frequency of this mutation in the general Ashkenazi population. The frequency of GD carriers among 308 TSD heterozygotes was 1:28 which is about half the expected $(P=0.03)$. These results indicate that carriers of both diseases do not possess additional evolutionary advantage over single mutation carriers. A reasonable interpretation of these findings is that one or both mutations have arisen relatively recently in different regions of Europe and have not yet reached genetic equilibrium.
\end{abstract}

Tay-Sachs disease (TSD) and Gaucher disease (GD) are glycolipid storage diseases caused by the impaired activity of the lysosomal enzymes hexosaminidase $A$ (EC 3.2.1.52) and glucocerebrosidase (EC 3.2.1.45), respectively. ${ }^{1,2}$ The incidence of both diseases is relatively high among the Jewish A shkenazi population, reaching a frequency of about 1:29 for TSD carriers and 1:16 for G D carriers. ${ }^{1,2}$

Two major hypotheses were proposed to account for this high frequency. O ne hypothesis suggests that genetic drift associated with a remarkable expansion of this population in Europe during the 16th to 19th centuries is the cause of the elevated carrier frequency. ${ }^{3}$ The other theory proposes that heterozygotes possess a

Correspondence, L eah Peleg, G enetic Institute, Sheba M edical Center, Tel Hashomer, 52621, I srael. Tel 9723 5302807; Fax: 97235302914

R eceived 28 July 1997; revised 150 ctober 1997; accepted 20 N ovember 1997 selective advantage over non-carriers. ${ }^{2}$ The advantage of carriers of lysosomal storage diseases has been attributed to the subclinical accumulation of the corresponding glycolipids in the lysosomes. ${ }^{4-6}$ If heterozygotes for each disease have indeed an evolutionary advantage, carriers of mutations for both diseases might be endowed with an additional advantage and their relative frequency in the population should increase. To test this hypothesis we studied the frequencies of the two predominant mutations of TSD and GD: the insertion mutation ( +1277TA TC) which accounts for about $83 \%$ of mutant TSD alleles, and the $1226 \rightarrow \mathrm{G}$ (1226G) mutation accounting for more than $70 \%$ of GD alleles ${ }^{1,2,7,8}$ We compared the frequency of the $G D$ $1226 \mathrm{G}$ carriers among A shkenazi heterozygotes of the TSD +1277TATC with that found in the general A shkenazi population. Carriers of TSD were identified through the nation-wide TSD screening and prevention programme. A Il participants completed a questionnaire 
Table 1 Frequency of GD heterozygotes among TSD carriers $^{b}$

\begin{tabular}{lccl}
\hline A shkenazi J ews & Total & G D carriers & Ratio \\
\hline G eneral population & 1503 & 108 & $1: 14$ \\
TSD heterozygotes & 330 & 13 & $1: 25.5$ \\
\hline
\end{tabular}

aCarriers of the $1226 \mathrm{G}$ mutation.

${ }^{b} \mathrm{C}$ arriers of the +1277TA TC mutation.

regarding ethnic extraction of their parents and grandparents.

A bout 11500 individuals with all four grandparents originating in Eastern or Central Europe were included. DNA samples of individuals who were diagnosed enzymatically as carriers ${ }^{9}$ were examined for the presence of the +1277TA TC insertion mutation, by heteroduplex formation; ${ }^{10} 308$ D NA samples carrying the TSD mutation were further analysed (anonymously) for the existence of the GD allele, 1226G, identified by the creation of the $X$ hol restriction site. ${ }^{11}$ The frequency of the $1226 \mathrm{G} \mathrm{GD}$ mutation among the general A shkenazi population was estimated by testing 1304 samples obtained through the routine screening service. The data presented in Table 1 show that the occurrence of GD carriers, bearing the $1226 \mathrm{G}$ mutation in the A shkenazi Jewish population screened by us (0.07), was similar to the recently published values ${ }^{1}$. However, among TSD heterozygotes, who carry the $(+1277$ TATC $)$, we found a significantly $\left(\chi^{2}=4.62\right.$, $P=0.03)$ lower frequency of the GD carriers (0.039). Thus, the occurrence of individuals who carry both mutations is significantly lower than expected from the combined frequencies of these two mutations in the A shkenazi population.

These results suggest that carriers of both diseases do not possess an evolutionary advantage over carriers of the single mutation. On the other hand, they do not rule out the possibility that heterozygosity to each disease confers such an advantage. The remarkable deficiency of double hetrozygotes may apparently indicate a disadvantage. $\mathrm{H}$ owever, this interpretation is not very likely since it would imply $50 \%$ lethality of double heterozygotes in the last generation, whereas there is no evidence suggesting that such individuals have any impairment of their health.

The most straightforward interpretation of our findings is that one or both mutations have arisen in the A shkenazi population relatively recently in different regions of Europe and have not yet reached genetic equilibrium.

\section{References}

1 Beutler E, Grabowski GA: G aucher disease. In: Scriver $C R, B$ eaudet $A L$, Sly W S and Valle D (eds). The M etabolic and M olecular Bases of Inherited D isease M CG raw-H ill, USA : 1995; pp 2641-2670.

2 Gravel RA, Clarke JTR, Kaback MM, Mahuran D, Sandhoff $K$ and Suzuki $K$ : The GM 2 gangliosidoses. In: Scriver CR, B eaudet $A L$, Sly WS and Valle D (eds). The $M$ etabolic and Molecular Bases of Inherited Disease. M cG raw-H ill, U SA : 1995; pp 2839-2882.

3 Goodman RM: Genetic Disorders among the Jewish People. Johns H opkins U niversity Press, Baltimore 1978.

4 Diamond JM: Jewish Lysosomes. Nature 1994; 368 : 291-292.

5 Zlotogora J, Ziegler M, Bach G: Selection in favor of lysosomal storage disorders?. A m J H um G enet 1988; 42: 271-273.

6 Zlotogora J: H igh frequencies of human genetic diseases: Founder effect with genetic drift or selection?. A m J M ed Gen 1994; 49: 10-13.

7 Beutler $E$, West C, G elbart T: Polymorphism in the human glucocerebrosidase gene. G enomics 1993; 12: 795-800.

8 K aback M M , Lim-Steele J, D abholkar D et al: Tay-Sachs disease carrier screening, prenatal diagnosis and the molecular era. JAMA 1993; 17: 2307-2315.

9 Peleg L, Karpati M, G azit E, R aas-R othschild A, G oldman $B$ : Mutations of the hexosaminidase $A$ gene in A shkenazi and non-A shkenazi Jews. Bioch Med M etab Biol 1994; 52: 22-26.

10 Shore S, M yerowitz R: A gel electrophoretic assay for detecting the insertion defect in A shkenazi Jewish carriers of Tay-Sachs disease. A nal B iochem 1990; 186: 179-181.

11 Beutler $E, G$ elbart T, West C: The facile detection of the nt 1226 mutation of glucocerebrosidase by 'mismatched' PCR . Clin Chim A cta 1990; 194: 161-166. 